\title{
Mental rotation of possible and impossible four-cornered toruses
}

\author{
RICHARD PRINGLE and THADDEUS M. COWAN \\ Kansas State University, Manhattan, Kansas 66506
}

\begin{abstract}
Possible and impossible four-cornered toruses were used as stimuli in a mental rotation paradigm. Rotation rates varied with number of "net changes in depth," a variable that closely corresponds with ratings of impossibility, rather than with formal distinctions between the possible and the impossible. Rotation rates were also affected by perimeter shapes but not in accord with perimeter complexity as defined by number of vertices. Experiment 2 attempted to separate net changes in depth from figure symmetry and found symmetry to be the more reliable predictor of rotation rates, suggesting that symmetry may underlie the influence of net changes of depth.
\end{abstract}

There have been two attempts to formulate rules for the systematic generation of impossible figures. Robinson and Wilson (1973) presented interesting variations of the three-stick clevis (Schuster, 1964) and pointed out that the construction of such figures takes advantage of the fact that a drawing of a clevis arm requires three lines whereas a cylindrical stick requires only two. Thus, any two rectilinear segments drawn in perspective can be made to terminate on five "sticklike" endings, though the figure is usually drawn terminating on three. This structural principle can, as the authors point out, be used to derive a multitude of impossible clevises or colonades where the clevis arms are n-sided and are made to terminate on circular or, alternatively, $\mathrm{k}$-sided endings where $\mathbf{k} \neq \mathbf{n}$. Additional variations result if the perspective of one end of the figure is made incompatible with the perspective of the other. This is a different element of impossibility, the same one involved in the figures described by Penrose and Penrose (1958) and more recently by Cowan $(1974,1977 \mathrm{a}, 1977 \mathrm{~b})$.

Cowan (1974) derived an algorithm whereby Penrose-type perspective figures with $\mathrm{n}$ sides can be generated systematically. All such figures are constructed from various combinations of the four corners $\left(M, N, Z, Z^{-1}\right)$ shown in Figure 1. Note that each corner is a unique perspective of a rightangled bend in an otherwise rectilinear structure. The rules of the algorithm dictate which corners can combine and in what order to form a possible figure.

Portions of this paper were presented at the annual meeting of the Psychonomic Society, St. Louis, 1976, and at the annual meeting of the Midwestern Psychological Association, Chicago, 1977. This research was conducted while the first author was supported by an NIMH experimental training grant (5T01MH08359) and while the second author was supported by an NSF grant (GBY/74-14014). Requests for reprints should be sent to Richard Pringle or Thaddeus M. Cowan, Department of Psychology, Kansas State University, Manhattan, Kansas 66506.
The corners are always combined by joining the origin of one corner with the terminus of another. The terminus and origin are arranged as described in Figure 1 so that their concatenation always proceeds in a clockwise direction. When a figure is constructed by joining the corners according to the arrows in Figure 1 (e.g., $\mathrm{M} \mathrm{M} \mathrm{Z}^{-1} \mathrm{Z}$ ), a possible figure is obtained. Care must be taken that the last corner and first corner also combine according to one of the arrows. If one goes against the direction of the arrows at least once, then an impossible figure results. The complete set of four-cornered torusespossible and impossible-are shown in Figure 2.

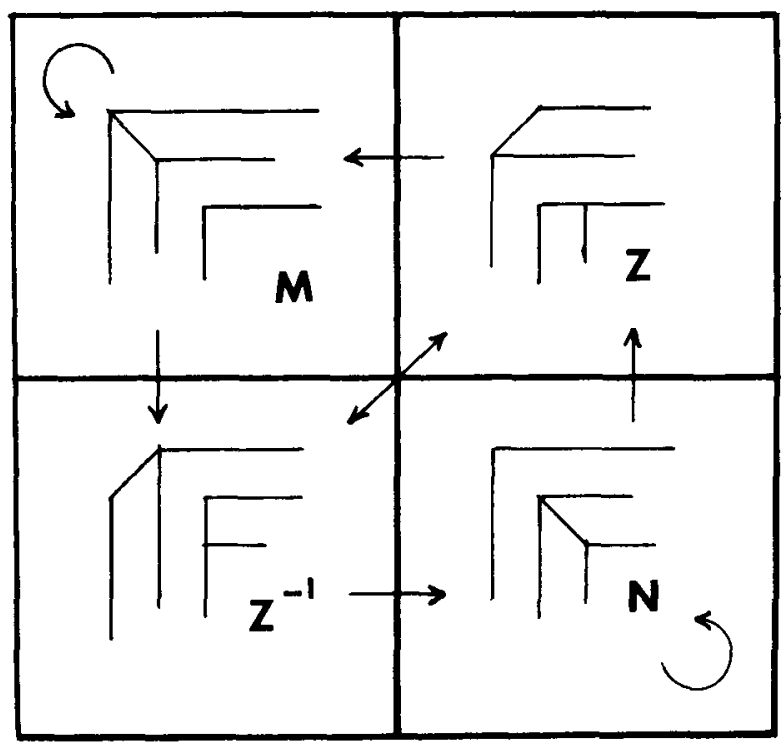

Figure 1. Cowan's (1974) algorithm for constructing possible figures. When the corners $M, N, Z$, and $Z^{-1}$ are combined in the direction of the arrows, possible figures result. Corners $M$ and $\mathbf{N}$ are self-composable. The end of the horizontal limb is the terminus, and the end of the vertical limb is the origin. 


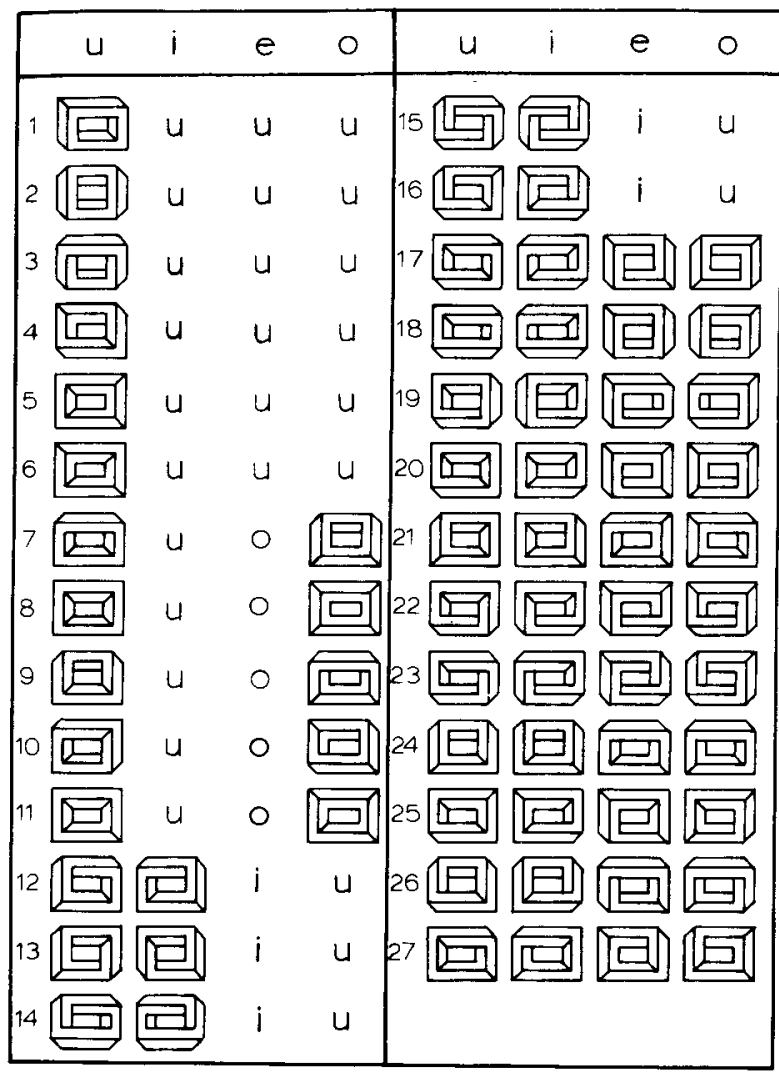

Figure 2. The complete set of four-cornered toruses $(u=$ unit figure; $\mathbf{i}=$ inverse; $e=$ everse; $0=$ obverse).

Figures labeled $\mathrm{u}$ represent the unit figures. Figures labeled $\mathrm{i}, \mathrm{o}$, and $\mathrm{e}$ are equivalent to the unit figures by simple transformations (versations): $\mathrm{i}=$ inverse (mirror image); $o=$ obverse $\left(180^{\circ}\right.$ rotation around the $\mathrm{x}$-axis); $\mathrm{e}=$ improper everse (mirror image of the obverse). Planar rotations are ignored. Note that some unit figures are unchanged by these operations (see Cowan, 1977a).

Cowan and Pringle (1978) have begun a series of studies designed to test the extent to which psychological and formal definitions of impossibility covary. One might expect, for example, that psychological possibility would be all or none, or perhaps that it would vary indirectly with the number of times the rules of Cowan's algorithm are broken in a figure's construction. Estimations of degree of possibility were collected on all four-cornered figures drawn normally and stereoptically. The estimations were made on a scale from 1 to 10 , where Torus 1 in Figure 2 was assigned a value of 10 . As expected, the four possible toruses $(1,2,7$, and 8$)$ were given high estimates of possibility. However, possibility was not an all-or-none phenomenon; some impossible figures were rated more possible than others. Nor was it the case that psychological impossibility corresponded to the number of times the rules of Cowan's algorithm were broken since, excluding the four possible figures, there was only a .01 correlation between the two. For the stereoptically drawn toruses the correlation was .25 but was also statistically insignificant.

What seemed to fit the data best was the number of "net changes in depth" a figure undergoes when examined in a consistent direction (e.g., clockwise). Each time that corners are combined inappropriately (i.e., in violation of Cowan's algorithm: Figure 1), a contortion is produced. Toruses 15 and 4 each have four such contortions, and they are illustrated in exploded forms in Figure 3. Such a contortion is meaningful if the side triplet produced by the corner combination is interpreted as undergoing a change in depth. The exploded side segments of Torus 15 seem to descend into the page as they are scanned in a clockwise direction, for a net change in depth of 4 . However, since the ever descending series returns to the original level, the intact figure is im-

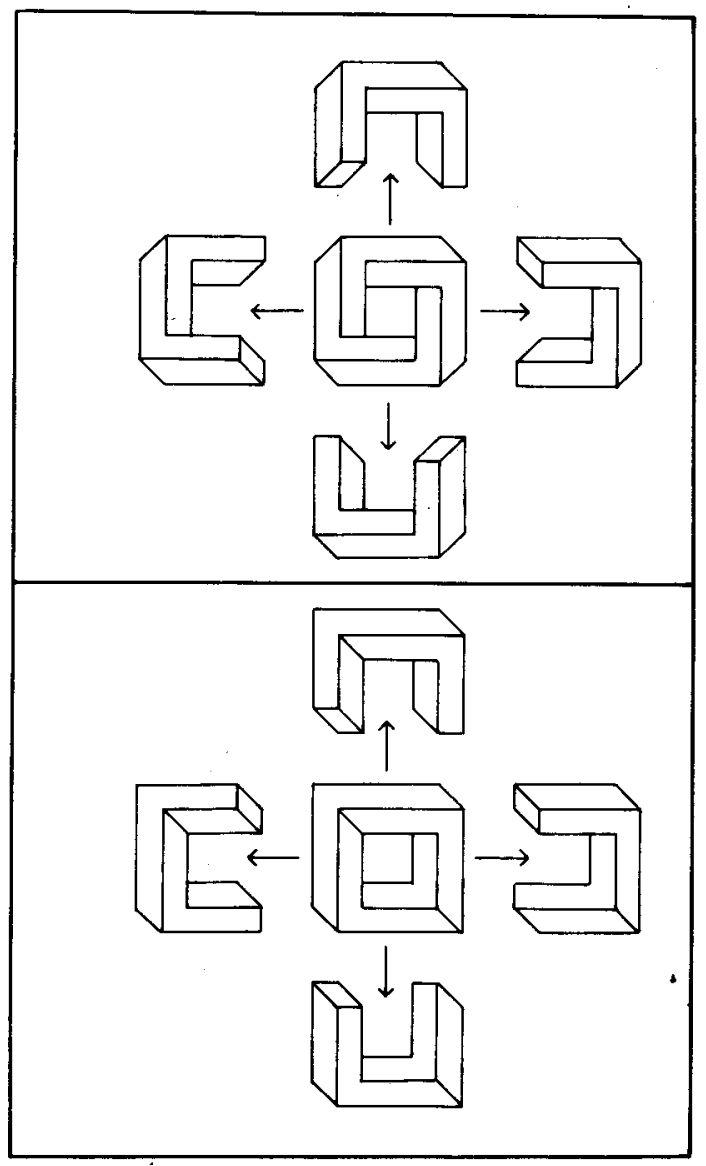

Figure 3. Torus 15 (top) and Torus 4 (bottom) with exploded side triplets showing different net change-in-depth values for two figures each with four contortions. 
possible in the same manner that the Penrose staircase is impossible. The greater the "net change in depth," the greater the element of this type of impossibility. Torus 4 also has four contortions, but two descend and two ascend for a net change of $0 .{ }^{1}$ There is a high negative correlation between judgments of possibility and the number of net changes in depth a figure undergoes. Excluding the four formally possible toruses, the correlation is -.67 for normally drawn toruses and -.78 for toruses drawn stereoptically. Thus, Torus 15 is consistently judged to be more impossible than Torus 4 .

The present study examines the extent to which this parameter determines the ability of subjects to manipulate torus figures cognitively-specifically if the rate of mental rotation (Shepard \& Metzler, 1971; Cooper \& Shepard, 1973; Cooper, 1976) of a torus figure is dependent on the net change in depth the figure undergoes. Cooper (1975) and Cooper and Podgorney (1976) found that figure complexity (as measured by number of vertices) of random shapes does not affect the rate of mental rotation, and, as. a consequence, they have argued that subjects rotate a holistic mental image at a constant rate. It may be the case, however, that subjects are incapable of incorporating an impossible figure into a single, holistic mental image. If the impossible toruses are interpreted three-dimensionally, then, by the laws of 3-space, certain parts of a figure imply other parts which are not in fact present. The composition does not fit existing schemata, and one might suspect that the mental image of such a figure would be unstable. If true, then, in a mental rotation paradigm, the effects would show up as a slower rotation rate for the impossible figures relative to the possible ones. But, of course, Cowan and Pringle (1978) have demonstrated that what subjects consider impossible in 3-space seems to be influenced by net changes in depth, more than by formal rules of composition in 3-space. Thus, an additional purpose of this study is to determine which description of impossibility (if either)-the psychological or the formal-determmines the rate and ability of subjects to mentally rotate four-cornered toruses.

\section{EXPERIMENT 1}

The stimuli to be rotated included nine toruses which were chosen from Figure 2 so as to conform to the 3 by 3 matrix shown in Figure 4. The three types of perimeter shapes (labeled " $P$ ") vary complexity in a manner similar to that used by Cooper. Perimeters 1 and 2 have six vertices; Perimeter 3 has eight. Within a particular perimiter shape level, the outside structure remains constant across the three possibility types (labeled “'T").
Unlike perimeter shape, variations along a "possibility" dimension would seem to manipulate complexity on a higher cognitive level than the "number of vertices" used by Cooper. Accordingly, such variations might affect the rate of mental rotation where perimeter complexity does not. The T1 figures are formally possible and undergo 0 net changes in depth. The T2 and T3 figures are formally impossible, the former undergoing 0 net changes in depth, the latter, 2 net changes. Any differences between the $\mathrm{T} 1$ and $\mathrm{T} 2$ figures will indicate an influence of mathematical or formal possibility. Differences between the T2 and T3 figures will indicate an influence of net changes in depth.

\section{Method}

\section{Subjects}

Four subjects were used. Three were undergraduates (female) recruited from psychology courses at Kansas State University and received partial course credit for their participation. One subject (male) was a graduate student volunteer. All subjects had normal or corrected vision.

\section{Stimuli}

Each torus subtended a $3.7^{\circ}$ visual angle in the center of a white circular background subtending $7.6^{\circ}$. Toruses were always presented in pairs in a two-channel tachistoscope. The center of the "standard" torus was presented $3.7^{\circ}$ to the right of midline; the center of the "comparison" was presented $3.7^{\circ}$ to the left. Each same torus pair consisted of a standard torus chosen from Figure 4 and a comparison that was a $0^{\circ}, 45^{\circ}, 90^{\circ}, 135^{\circ}$, or $180^{\circ}$ clockwise rotation of the standard.

A variation of the standard procedure for constructing different stimuli by pairing standards with their mirror reflections (e.g., Cooper \& Shepard, 1973; Shepard \& Metzler, 1971) was followed here. Six of the nine toruses of Figure 4 are symmetrical, and a mirror reflection of a symmetrical figure is always a simple planar rotation of the original figure. Thus, if the correct response is to be "different," then for a symmetrical torus, the comparison must be structurally different from the standard. Such structurally different stimuli were constructed from the toruses of Figure 4.

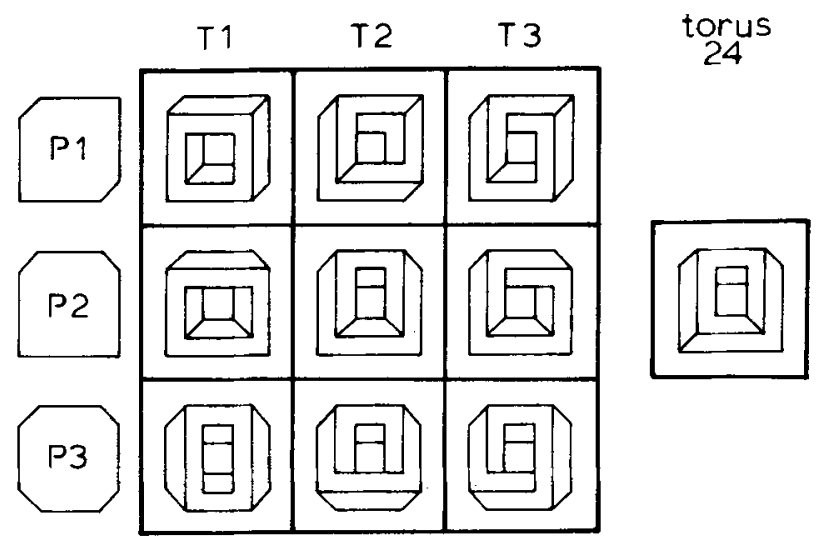

Figure 4. The matrix of stimuli used in Experiments 1 and 2. Torus 24 was added in Experiment 2. (T1, T2, and T3 refer to various types of figure possibility; P1, P2, and P3 refer to perimeter shapes.) 
In order to insure that these "structurally different" stimuli retained a measure of similarity, each standard and comparison pair had identical perimeter shapes.

If structural differences are too great, subjects might be able to perform a match without mental rotation. To minimize the use of such a strategy, a second class of different stimuli was included. For these stimuli, the comparison was always a mirror-reflected version of the standard, but neither the standard nor comparison were taken from Figure 4. They were taken from the asymmetrical toruses in Figure 2 that were not otherwise included in Figure 4 (i.e., Toruses 12, 16, 17, 18, 19, 20, 21, 23, 24, 25, 26, and 27). Overall, $20 \%$ of the different stimuli were of the structurally different class, and $80 \%$ were of the mirror-reflection class. It is important to note that the test of the adequacy of this procedure is simply whether or not subjects mentally rotate the toruses. To the extent that there are appropriate response time differences across the various rotations for same trials, the procedure can be said to have been a sufficient inducement of mental rotation.

\section{Apparatus}

The standard and comparison of each torus pair were presented simultaneously via a two-channel tachistoscope under an illumination of $67 \mathrm{~mL}$. To initiate a trial, the experimester closed a switch which sounded a 500 -msec warning tone. The torus pair was presented $1 \mathrm{sec}$ after the termination of the tone. The subject pressed a key to indicate when he or she had decided if the toruses were same or different, and simultaneous with pressing the key, the subject responded "same" or "different" verbally. A Standard 60-sec clock began timing the response interval when the toruses were presented and was terminated by the keypress. Trials wherein the subject's verbal response was judged to occur after the keypress were repeated later in the session, and the subject was reminded that both responses should occur simultaneously.

\section{Procedure}

The complete experimental design included four factors: three possibility types, three perimeter shapes, five extents of rotation, and four replications. The five extents of rotation were $0^{\circ}, 45^{\circ}$, $90^{\circ}, 135^{\circ}$, and $180^{\circ}$. Due to Torus 2 , the experimental design is actually incomplete across all five rotations. Torus 2 has two axes of symmetry, and consequently, the $135^{\circ}$ and $180^{\circ}$ rotations are redundant with its $45^{\circ}$ and $0^{\circ}$ rotations, respectively. Consequently, the experimental design is complete only across $0^{\circ}, 45^{\circ}$, and $90^{\circ}$ rotations.

Each subject participaled in five 1-h sessions of 117 trials each. The first session was considered practice, and the first 27 trials of each session were warm-up trials involving toruses of Figure 2 that were not otherwise included in the experiment. Nine warm-up stimuli pairs (five same and four different) were presented three times in random orders with appropriate counterbalancing of extent of rotation and the orientation of the standard torus. Ninety experimental trials followed the 27 warm-up trials and consisted of 45 same and 45 different pairs presented in one of four random orders.

The 45 same stimuli consisted of the nine toruses of Figure 4 presented in each of the five rotation conditions. The orientation of the standard toruses (horizontal, vertical, and left and right oblique) was counterbalanced across the four random orders of presentation. The $\mathbf{4 5}$ different stimuli consisted of 9 structurally different and 36 mirror-reflected pairs. Rotations for these stimuli were defined in terms of arbitrary partial congruences.

Only correct responses to same stimuli were included in the analysis, and all incorrect responses to the same stimuli were repeated (with filler trials) at a later point in the session.

\section{Results}

Mean error rate for same trials was $4.0 \%$ and varied from $0 \%$ to $10 \%$ for individual subjects.
Mean error rate for different trials was $6.0 \%$ and varied from $0 \%$ to $8.3 \%$.

Reaction times to correctly respond "same" pooled across all four subjects are plotted as a function of extent of rotation in Figure $5 \mathrm{a}$. Since the $135^{\circ}$ and $180^{\circ}$ rotations of Torus 2 are undefined, only the first three extents of rotation are included. It is apparent from Figure 5a that the reaction times increased in a linear manner. A regression analysis attributed $98.9 \%$ of the variance to the linear component. This linearity is in agreement with other mental rotation studies (e.g., Shepard \& Metzler, 1971) and suggests that in order to make an identity match of four-cornered toruses, subjects mentally rotate (at a constant rate) one torus into congruence with the other.

Analyses of variance were performed on each subject individually and were based on the first three extents of rotation. ${ }^{2}$ There were significant main effects of degree of rotation for three subjects $[\mathrm{S} 1: \mathrm{F}(2,81)=43.83, \mathrm{p}<.01 ; \mathrm{S} 2: \mathrm{F}(2,81)=18.38$, $\mathrm{p}<.01 ; \mathrm{S} 4: \mathrm{F}(2,81)=13.82, \mathrm{p}<.01]$, and the fourth approached significance $[S 3: F(2,81)=2.83$, $\mathrm{p}<.07]$. A trend analysis revealed significant linear components for all subjects, and all residual components were insignificant. The mean rotation rate across all subjects using the least squares method was $67 \mathrm{deg} / \mathrm{sec}$, which is similar to the $60 \mathrm{deg} / \mathrm{sec}$ obtained by Shepard and Metzler (1971) for their perspective figures.

\section{Perimeter Shape}

The reaction times pooled across subjects are plotted against extent of rotation for the three perimeter shapes in Figure 5b. There were significant main effects of perimeter shape for three subjects, and all three were due primarily to the longer RTs associated with $\mathrm{P} 1$ than with $\mathrm{P} 2$ or $\mathrm{P} 3$. A perimeter

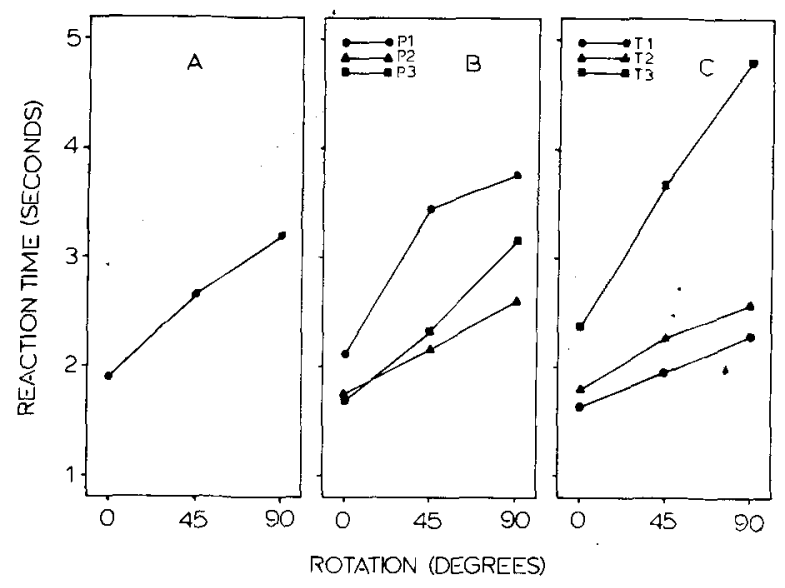

Figure 5. Reaction times from Experiment 1 as a function of: (A) extent of rotation; (B) extent of rotation for each perimeter shape (P1, P2, and P3); (C) extent of rotation for each possibility type (T1, T2, and T3). 
effect produced by number of vertices would predict longer RTs to P3 than to P1 or P2, and in this respect these results are consistent with Cooper (1975), where no main effects of number of vertices was found.

The "savings" associated with the P2 and P3 perimeters did not give rise to faster mental rotation. There was only one subject (S2) who showed a perimeter shape by extent of rotation interaction, and it was due to the faster rotation of the $\mathrm{P} 2$ figures relative to the $\mathrm{P} 1$ and $\mathrm{P} 3$ figures. This effect was also inconsistent with an effect of number of vertices, since the $\mathrm{P} 1$ and $\mathrm{P} 3$ toruses have six and eight vertices, respectively. Thus, as in Cooper's random shape study, number of vertices did not affect the rate of mental rotation.

\section{Possibility Type}

The mean reaction times across all subjects are plotted against extent of rotation for the three possibility types in Figure 5c. The main effect of possibility type was significant for all subjects and was primarily due to the slower RTs associated with the 2 net-change-in-depth toruses (T3). For all subjects, the impossible 0-net-change toruses (T2) elicited slower RTs than the possible 0-net-change toruses (T1), but this difference reached significance for only one subject (S1). In contrast, the differences between the 0 - and 2-net-change impossible toruses was significant for all subjects [S1: $\mathrm{F}(1,81)=18.82, \mathrm{p}<.01$; S2: $F(1,81)=23.87, p<.01 ; S 3: F(1,81)=16.32$, $\mathrm{p}<.01 ; \mathrm{S} 4: \mathrm{F}(1,81)=34.80, \mathrm{p}<.01]$. The direction of these differences was consistent: $\mathrm{T} 1<\mathrm{T} 2<\mathrm{T} 3$.

In addition to the main effect of possibility type, there were significant Possibility Type by Extent of Rotation interactions for two of the four subjects, and a third approached significance [S1: $F(4,81)=$ $4.68, \mathrm{p}<.01 ; \mathrm{S} 2: \mathrm{F}(4,81)=2.06, \mathrm{p}<.10 ; \mathrm{S} 4$ : $F(4,81)=3.40, p<.05]$. This interaction pooled across subjects is shown in Figure $5 c$. In each instance, the interaction was due to the greater slope (slower rotation rate) of the impossible 2-net-change toruses (T3) relative to the 0-net-change-in-depth toruses (T1 and T2) [S1: $\mathrm{F}(2,81)=5.81, \mathrm{p}<.01$; $\mathrm{S} 2: \mathrm{F}(2,81)=3.89, \mathrm{p}<.05 ; \mathrm{S} 4: \mathrm{F}(4,81)=6.47$, $\mathrm{p}<.01]$. There were no slope differences between the possible and impossible 0-net-change toruses. On average, the $\mathrm{T} 1$ and $\mathrm{T} 2$ figures were rotated at $113 \mathrm{deg} / \mathrm{sec}$. The T3 figures, on the other hand, were rotated at $37 \mathrm{deg} / \mathrm{sec}$.

The rotation rate differences seem to follow net changes in depth rather than formal variations of possibility. The fact that some toruses are impossible of itself makes little difference in the rate of mental rotation. Thus, in contrast to Cooper (1975) and Cooper and Podgorny (1976), the present study demonstrates that complexity can influence the rate of mental rotation when that complexity is of a high order.

A study by Corballis and Roldan (1975) suggests an alternative to the net-changes-in-depth explanation. They found that in order to decide if dot patterns were symmetrical around a predesignated axis, subjects mentally rotated the axis back to vertical. In one of three experiments, the symmetrical patterns were rotated faster than the asymmetrical patterns. The other two experiments found no rate differences. Though the results are equivocal, they suggest that figure complexity in terms of symmetry may affect rotation rates. In this regard, it is interesting to note that the 0-net-change-in-depth toruses used here are all symmetrical whereas the 2-netchange-in-depth toruses are not. It could be the case that symmetrical toruses are rotated faster than asymmetrical toruses.

There is only one figure (Torus 24) which has 0 net changes in depth and is asymmetrical. If, as the Corballis and Roldan study suggests, symmetry affects rate of rotation, then the responses to Torus 24 should mimic those given to the T3 toruses since they are asymmetrical. To the extent that net change in depth is the critical factor, the responses should mimic those given to the T2 toruses, since they undergo 0 net changes in depth. Of course, if the responses to Torus 24 fall somewhere between those of the T2 and T3 toruses, one might argue that both symmetry and net changes in depth are important.

\section{EXPERIMENT 2}

So as to examine the effects of torus symmetry, Experiment 2 replicated Experiment 1 with three modifications: (1) Torus 24 was added to the matrix of nine stimuli used in the first experiment; (2) the procedure was changed to enable both the analysis of different trials and the completion of the experimental design across all five extents of rotation; (3) six subjects were used so as to allow a group analysis and, accordingly, make possible an examination of the effects of replications on the relative rotation rates of the various classes of figures. It could be the case that differences in rate of rotation diminish with practice.

\section{Subjects}

\section{Method}

Subjects were six undergraduates (one male and five female) recruited from general psychology courses at Kansas State University. All received partial course credit for their participation.

\section{Stimuli}

Torus 24 was added to the matrix of stimuli in Figure 4. As in Experiment 1, each same torus pair consisted of a standard torus chosen from Figure 4 and a comparison that was $0^{\circ}, 45^{\circ}$, 
$90^{\circ}, 135^{\circ}$, or $180^{\circ}$ clockwise rotation of the standard. Each different torus pair consisted of a standard from Figure 4 and a comparison (also chosen from the toruses of Figure 4 or their inverse, obverse, or everse transformations) that (1) had the same perimeter shape as the standard and (2) had $0,1,2$, or 3 corners in common with the correct rotation of the standard. If subjects rotate the figures piecemeal by a serial process, then one might expect that the more matched corners there are between the comparison and the mentally rotated standard, the longer on average the rotation-decision process would take (see Sternberg, 1969a, 1969b).

\section{Procedure}

As in Experiment 1, the stimuli were presented in pairs via a two-channel tachistoscope. However, prior to each trial, the experimenter would name one of the five rotations. Thereafter, the subject initiated the trial by pressing a foot switch that simultaneously started the clock. The subject pressed a right-hand key if the figures were related by the rotation named and a left-hand key if they were not so related. The comparison could have $0,1,2$, 3 , or 4 corners in common with the correct rotation of the standard. Note that in this procedure it is irrelevant that a $45^{\circ}$ rotation happens to be equivalent to the $135^{\circ}$ rotation, or that $0^{\circ}$ happens to equal $180^{\circ}$. Also, the procedure allows for the systematic treatment of different trials, since presumably the rotation performed would always be equivalent to the rotation named by the experimenter, avoiding the problem of defining extent of rotation in terms of partial congruences.

Each of the 10 toruses was paired twice (same and different), with each of the five rotation conditions for a total of 100 trials/ replication. Each session consisted of 20 warm-up trials (counterbalanced for same-different condition, figures, and extent of rotation) and one experimental replication. Each subject was run in five sessions for a total of five replications, the first of which was considered practice. For different trials, the number of matching corners between the comparison and the mentally rotated standard was confounded with the Perimeter Shape by Possibility Type by Extent of Rotation by Replication interaction. However, this confounding was randomized across rotation, figures, and subjects,

The complete experimental design consisted of 6 subjects, 3 perimeter shapes, 3 possibility types, 5 extents of rotation, and 2 conditions (same-different). In addition, Torus 24 was included. As in Experiment 1, the orientation of the standard (vertical, horizontal, and left and right oblique) was counterbalanced across four random orders of presentation. All incorrect trials were repeated later within the same session.

\section{Results}

Pooled reaction times as a function of extent of rotation for both same and different trials are shown in Figure 6. The most striking feature of these graphs is the apparent drop at $180^{\circ}$. All subjects showed the effect. It became obvious in the course of the experiment that such an effect was present, and in an attempt to explain the phenomenon all subjects after the end of the final session were informally questioned about their strategy. All six subjects described some type of mental rotation process. Next, they were asked if they had used the same strategy for all five rotations. Five of the six subjects described a different process for the $180^{\circ}$ rotation than for the other four rotations. Rather than a planar rotation, they described a sort of "flipping", through 3-space. When it was pointed out that such

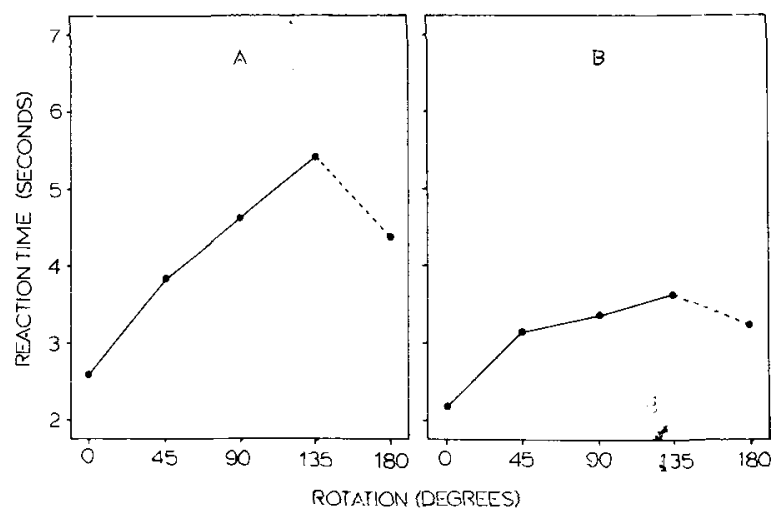

Figure 6. Reaction times from Experiment 2 as a function of extent of rotation for (A) same trials and (B) different trials.

a transformation would produce the mirror image of the correct rotation, the subjects expressed some surprise. Only S4 verbally reported nothing unusual about the $180^{\circ}$ rotation; however, his data show the same effect as the other subjects.

Because the primary purpose of this experiment was to examine differences in linear slopes (rotation rates), the $180^{\circ}$ points were dropped from further analysis.

The overall error rate was $5.3 \%$. Separate analyses of variance were performed for same and different trials, and they excluded Torus 24 .

\section{Same Trials}

Reliable main effects were obtained for extent of rotation $[F(3,15)=26.93, p<.01]$, possibility type $[F(2,10)=41.72, p<.01]$, and perimeter shape $[F(2,10)=25.32, p<.01]$. Both perimeter shape and possibility type interacted with extent of rotation $[F(6,30)=4.10, p<.01$, and $F(6,30)=6.35$, $p<.01$, respectivelyl. There was no effect of replications excepting an interaction with possibility type $[F(6,30)=2.99, p<.05]$. A trend analysis revealed a significant linear component $[\mathrm{F}(1,5)=32.73$, $\mathrm{p}<.01]$, and the residual component failed to reach statistical significance $[F(2,10)=2.26]$. The linear component accounted for $98.4 \%$ of the variance. Mean rotation rate using the least squares method was $48 \mathrm{deg} / \mathrm{sec}$.

Perimeter shape. Pooled reaction times as a function of perimeter shape and extent of rotation are shown in Figure 7a. As in Experiment 1, longer reaction times were associated with the $P 1$ figures rèlative to the P2 and P3 figures $[F(1,5)=40.44, p<.01]$. In this instance, however, the savings were also reflected in rotation rates. Orthogonal analyses of the Perimeter Shape by Extent of Rotation interaction attributed the effect to the slower rotation of the $P 1$ figures $[F(3,15)=5.79, p<.01]$. There was no difference between the $\mathrm{P} 2$ and $\mathrm{P} 3$ rotation rates. The 


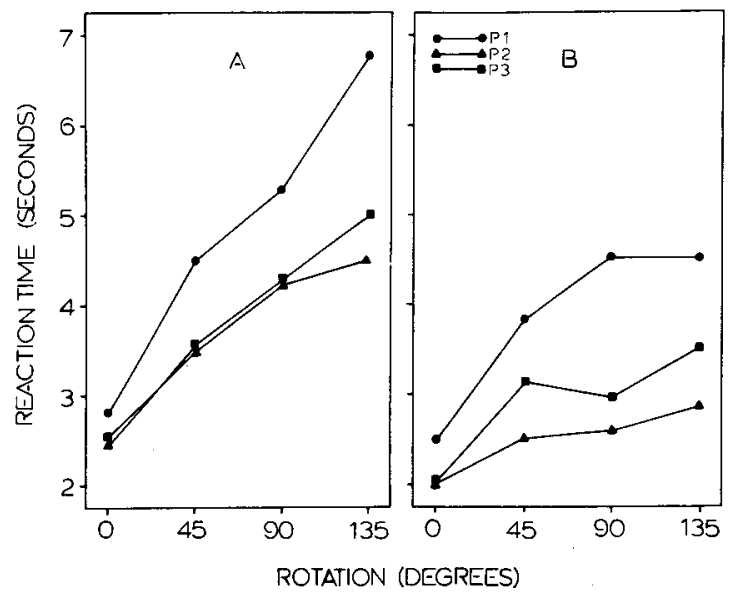

Figure 7. Reaction times from Experiment 2 as a function of extent of rotation for each perimeter shape (P1, P2, and P3) for (A) same trials and (B) different trials.

$\mathrm{P} 1$ figures were rotated at $36 \mathrm{deg} / \mathrm{sec}$, while the $\mathbf{P} 2$ and P3 figures were rotated $58 \mathrm{deg} / \mathrm{sec}$. These differences in overall reaction times and in rotation rates are not in the expected direction in regard to complexity of perimeter shape as defined by the number of vertices, and accordingly, these results are consistent with those of Cooper (1975).

Possibility type. Pooled reaction times as a function of possibility type and extent of rotation are shown in Figure 8a. The T3 figures are associated with slower reaction times than the $\mathrm{T} 1$ and $\mathrm{T} 2$ figures $[F(1,5)=45.31, p<.01]$. The difference between the $T 1$ and $T 2$ figures was also reliable $[F(1,5)=7.75$; $\mathrm{p}<.05$ ]. In addition, just as in Experiment 1, the T3 figures were rotated more slowly than the T1 and $T 2$ figures $[F(3,15)=10.06, p<.01]$. There was no rotation rate difference between the $T 1$ and $\mathrm{T} 2$ figures $[\mathrm{F}(3,15)=0.54]$. The $\mathrm{T} 3$ figures were rotated at $36 \mathrm{deg} / \mathrm{sec}$, whereas the $\mathrm{T} 1$ and $\mathrm{T} 2$ figures were both rotated at $56 \mathrm{deg} / \mathrm{sec}$.

Torus 24. Reaction times for Torus 24 is shown along with the other P2 figures in Figure 9. The average rate of rotation of Torus 24 was $34 \mathrm{deg} / \mathrm{sec}$. Clearly, it falls in line more closely with the T3 figures than with the T2 figures. Since Torus 24 is a 0 -net-change-in-depth figure, the results suggest that figure symmetry is more important than net levels of depth in determining rate of rotation. ${ }^{3}$

\section{Different Trials}

Reliable main effects were obtained for extent of rotation $[F(3,15)=24.36, p<.01]$, possibility type $[F(2,10)=25.81, p<.01]$, and perimeter shape $[\mathrm{F}(2,10)=26.59, \mathrm{p}<.01]$. Both perimeter shape and possibility type interacted with extent of rotation $[F(6,30)=8.56, \mathrm{p}<.01$, and $F(6,30)=4.16$, $\mathrm{p}<.05$, respectively]. All orthogonal comparisons mirrored those in the same trial analysis except in two instances. An analysis of the Perimeter Shape by Extent of Rotation interaction revealed reliable rotation rate differences between the P2 and P3 figures $[F(3,15)=5.05, p<.01]$, as well as between the $P 1$ and $P 2$ figures $[F(3,15)=10.40, p<.01]$. In addition, an analysis of trend indicated a reliable nonlinearity across extent of rotation for different trials

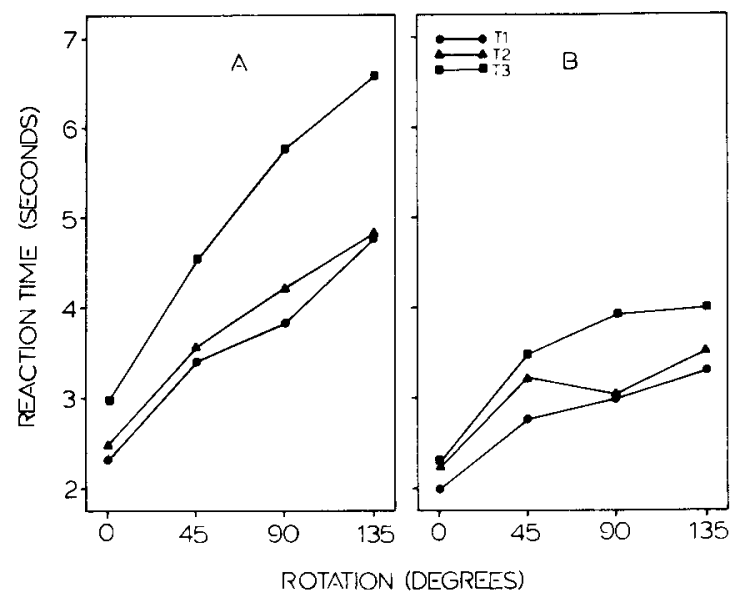

Figure 8. Reaction times from Experiment 2 as a function of extent of rotation and possibility type (T1, T2, and $T 3$ ) for (A) same trials and (B) different trials.

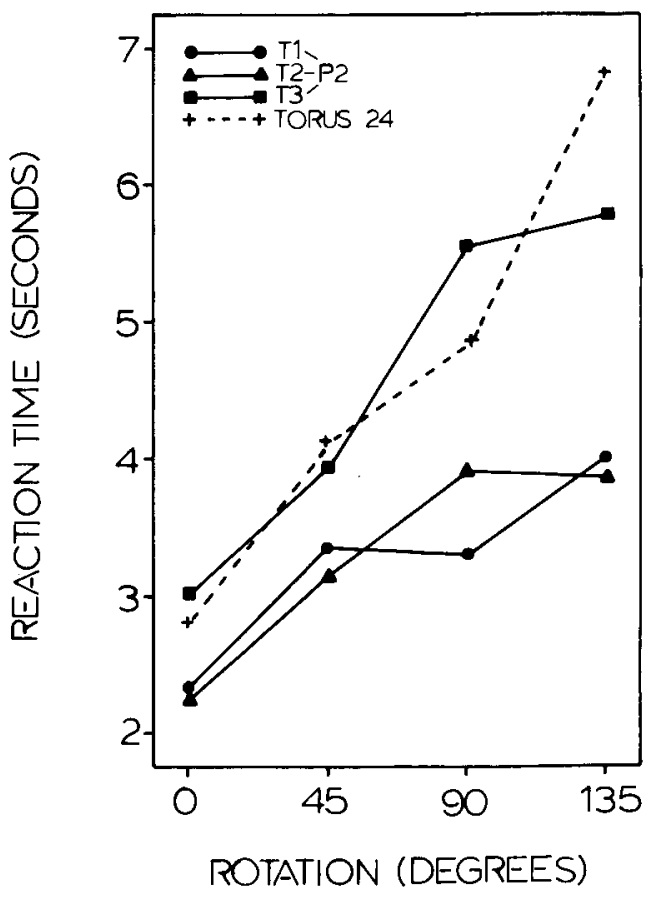

Figure 9. Reaction times from Experiment 2, for the P2 figures only, as a function of extent of rotation and possibility type (T1, T2, and T3). Torus 24 is indicated by dotted line. 
$[F(2,10)=8.54, p<.01]$. Inspection of Figure $6 b$ reveals that the $0^{\circ}$ rotation point is low relative to the $45^{\circ}, 90^{\circ}$, and $135^{\circ}$ points, and probably indicates that knowing beforehand that no transformation was required had a facilitory effect on processing the $0^{\circ}$ rotation. Note that this tendency is also present for the same trials (Figure 6a), but did not give rise to a statistically significant nonlinearity.

If one assumes that the $45^{\circ}, 90^{\circ}$, and $135^{\circ}$ rotation points are the best indicators of slope (rotation rate), then an additional difference between the same and different conditions becomes apparent in Figure 8b. There are no slope differences due to possibility type for the different trials. This result is not unreasonable, however, since for different trials the standard and comparison were not always of the same possibility type. Possibility types in Figure $8 b$ refer to the possibility types of the standard. If one assumes that subjects will rotate that figure that has 0 net changes in depth (regardless if it is the standard or comparison), then only those instances where both figures are 2-net-change-in-depth toruses will show a slower rate of rotation. This proved to be the case, as is shown in Figure 10. When both standard and comparison were T3 figures, the rotation rate was $41 \mathrm{deg} / \mathrm{sec}$. When at least one was a 0 -net-change figure, the rotation rate was $101 \mathrm{deg} / \mathrm{sec}$.

Standard and comparison pairs were always of identical permiter shapes, and consequently one would expect the perimeter-shape rate differences found for same trials to also be present for different trials. This conjecture proved true, as shown in Figure $7 \mathrm{~b}$. The $\mathrm{P} 1$ figures were rotated at $55 \mathrm{deg} / \mathrm{sec}$, whereas the P2 and P3 figures were rotated at 155 and $83 \mathrm{deg} / \mathrm{sec}$, respectively.

Effects of the number of matching corners between the comparison and the mentally rotated standard were unsystematic and suggest that if subjects were, in fact, rotating the toruses piecemeal (and the difference in rates of rotation between the same and different trials suggest they were), the "unit" of rotation was not individual corners.

Individual subject analyses parallel the group analyses reported here, with the single exception that there was a tendency for the Possibility Type by Extent of Rotation interactions not to reach statistical significance. All rotation rate differences were in the appropriate direction, however.

\section{CONCLUSIONS}

Both of these experiments agree on two principle points. (1) Figure complexity does affect rates of rotation, but number of vertices is not a sufficient inducement of such differences. When complexity is of a high order (e.g., net changes in depth, figure symmetry), differences in rotation rates follow.

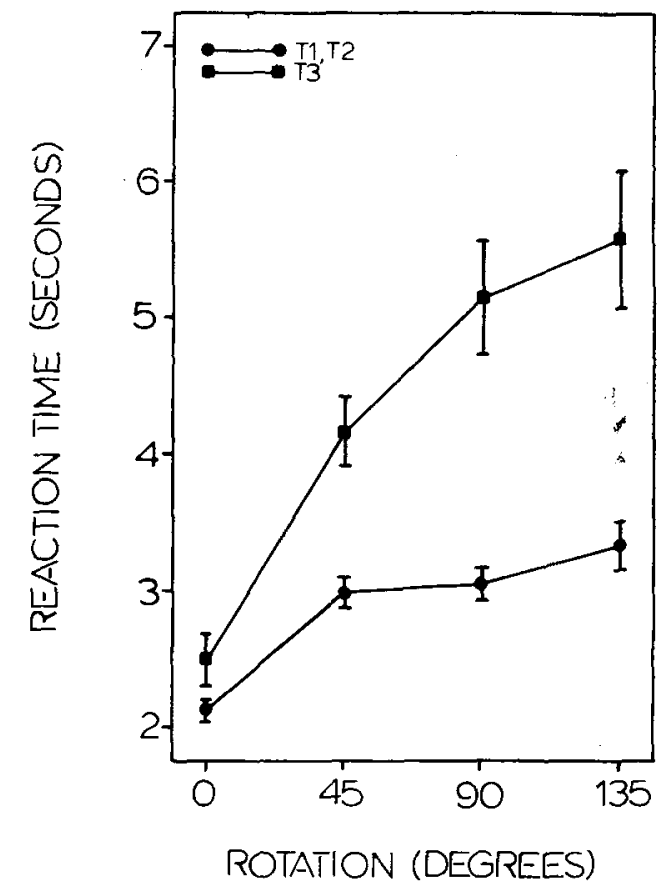

Figure 10. Reaction times from Experiment 2 as a function of extent of rotation for standard-comparison different pairs, wherein both figures underwent 2 net changes in depth (top curve) and at least one figure underwent 0 net changes in depth (bottom curve). Standard errors around each point are shown.

Perimeter shape can also affect rotation rate as shown in Experiment 2. Figures having the $P 2$ and $P 3$ perimeters are more easily manipulated than those having the $P 1$ perimeter. It is interesting to note that $P 1$ perimeters have a diagonal symmetry whereas $\mathrm{P} 2$ and $\mathrm{P} 3$ perimeters both have a perpendicular symmetry, and this difference may account for the observed reaction time differences. (2) The psychological continuum of possibility (corresponding roughly to net changes in depth) is a better predictor of rotation rate differences and reaction times in general than the formal continuum of possibility as defined by Cowan's algorithm (1974). That a figure can or cannot exist in 3-space seems to make little difference except as it agrees with the net levels of depth hierarchy.

In addition, Experiment 2 has suggested that figure symmetry may be the principle factor by which net level of depth becomes such a powerful variable. The two dimensions can never be completely' separated, however, because ultimately net levels of depth can be reduced to a type of symmetry between corners. One method of determining the net changes in depth a figures undergoes is by computing the absolute value of the number of $\mathrm{Z}$ corners minus the number of $Z^{-1}$ zorners. By this method, net depth change is dependent entirely on $Z$ and $Z^{-1}$ corners. A $Z^{-1}$ corner is the mirror image of a $\mathrm{Z}$ corner. 
Thus, when these two corners occur in unison, there is both symmetry and no net change in depth. If one occurs without the other, there is both asymmetry and a change in depth. Torus 24 has one $Z$ and one $\mathrm{Z}^{-1}$ corner, and therefore undergoes 0 net changes in depth. It obtains its overall figure asymmetry not from the Z-type corners, but rather from having one $\mathbf{M}$ and one $\mathbf{N}$ corner. One might, in this case, want to speak of a partial symmetry-exclusive to Z-type corners. In this sense, then, symmetry and net changes in depth are forever confounded. Even for toruses having other than four sides, changes in depth can still be defined in terms of $\mathrm{Z}-\mathrm{Z}^{-1}$, and thus in terms of symmetry between the Z-type corners.

It is important to note that these rotation rate differences do not seem to diminish with time-at least not across the five sessions examined herein. There were few effects of replications. If anything, the differences become more pronounced in that the linear functions become smoother.

\section{REFERENCES}

Cooper, L. A. Mental rotation of random two-dimensional shapes. Cognitive Psychology, 1975, 7, 20-43.

Cooper, L. A. Demonstration of a mental analogue of an external rotation. Perception \& Psychophysics, 1976, 19. 296-302.

Cooper, L. A., \& Podgorny, P. Mental transformations and visual comparison processes: Effects of complexity and similarity. Joumal of Experimental Psychology: Human Perception and Performance, 1976, 2, 503-514.

Cooper, L. A., \& Shepard, R. N. Chronometric studies of the rotation of mental images. In W. G. Chase (Ed.), Visual information processing. New York: Academic Press, 1973.

Corballis, M. C., \& Roldan, C. E. Detection of symmetry as a function of angular orientation. Journal of Experimental Psychology: Human Perception and Performance, 1975, 1. 221-230.

Cow AN T. M. The theory of braids and the analysis of impossible figures. Journal of Mathematical Psychology, $1974,11,190-212$.

Cowan, T. M. Organizing the properties of impossible figures. Perception, 1977, 6. 41-56. (a)

Cowan, T. M. Supplementary report: Braids, side segments, and impossible figures. Journal of Mathematical Psychology, 1977, 16, 254-260. (b)
Cowan, T. M., \& Pringle, R. An investigation of the cues responsible for figure impossibility. Journal of Experimental Psychology: Human Perception and Performance, 1978, 4. 112.120

Penrose, L. S., \& Penrose, R. Impossible objects: A special type of illusion. British Journal of Psychology, 1958, 49, 31-33.

Robinson, J. O., \& Wilson, J. A. The impossible colonade and other variations of a well-known figure. British Journal of Psychology, 1973, 64, 363-365.

Schuster, D. H. A new ambiguous figure: A three-stick clevis. American Journal of Psychology, 1964. 77, 673.

ShePard, R. N., \& Metzler, J. Mental rotation of threedimensional objects. Science, 1971, 171, 701-703.

STERnBERG, S. Memory-scanning: Memory processes revealed by reaction-time experiments. American Scientist, 1969, 57, 421.457. (a)

STERnBERG, S. The discovery of processing stages: Extensions of Donder's method. Acta Psychologica, 1969, 30, 276-315. (b)

\section{NOTES}

1. An equivalent method of determining the net changes in depth a figure undergoes is to simply take the absolute value of the difference between the number of $Z$ corners and number of $\mathrm{Z}^{-1}$ corners in the torus (i.e., $/ Z-Z^{-1} /$ ). Torus 24 , for example, has four $Z^{-1}$ corners or $/ 0-4 /=4$ net changes in depth. Torus 4 has two $\mathrm{Z}^{-1}$ and two $\mathrm{Z}$ corners or $/ 2-2 /=0$ net changes in depth.

2. Analyses including all five extents of rotation were in agreement with the results reported here, with the exception that two subjects ( $\mathrm{S} 2$ and $\mathrm{S} 4$ ) showed significant nonlinearities. The functions leveled off (S4) or declined slightly (S2) at the $180^{\circ}$ rotation points. Whatever the cause of the nonlinearity, it had disappeared by the final replication.

3. Another possible interpretation of these results was suggested by a referee. In the T3 toruses and in Torus 24 , the two permissible corner junctions are always adjacent to one another. In the $T 2$ toruses, the permissible corner junctions are always on opposite sides of the torus. It is possible that this factor, rather than symmetry, is determining rotation rates. We have tested this notion using the four P2 figures of Experiment 2 as well as Torus 12 from Figure 2. Torus 12 is impossible, asymmetrical, with its permissible corner junctions on opposite sides of the torus. It was rotated even more slowly than either the P2 T3 torus or. Torus 24. This strongly suggests that "adjacency of permissible junction" is not an important factor.

(Received for publication October 5, 1977; revision accepted April 20,1978.) 